\title{
Fast image watermarking based on signum of cosine matrix
}

\author{
Ferda Ernawan ${ }^{1}$, Prajanto Wahyu Adi², Siau-Chuin Liew ${ }^{1}$, Eko Adi Sarwoko ${ }^{2}$, Edy Winarno ${ }^{3}$ \\ ${ }^{1}$ Faculty of Computing, College of Computing and Applied Sciences, Universiti Malaysia Pahang, Pekan, Malaysia \\ ${ }^{2}$ Department of Informatics, Faculty of Science and Mathematics, Universitas Diponegoro, Central Java, Indonesia \\ ${ }^{3}$ Department of Information Technology, Faculty of Information Technology, Universitas Stikubank, Central Java, Indonesia
}

\begin{tabular}{|c|c|}
\hline Article Info & ABSTRACT \\
\hline Article history: & In the field of image watermarking, the singular value decomposition has good \\
\hline Received Mar 9, 2021 & $\begin{array}{l}\text { imperceptibility and robustness, but it has high complexity. It divides a host } \\
\text { image into matrices of U, S and V. Singular matrix } S \text { has been widely used }\end{array}$ \\
\hline Revised Nov 11, 2021 & for embedding and extracting watermark, while orthogonal matrices of $U$ and \\
\hline Accepted Dec 2, 2021 & $\begin{array}{l}\mathrm{V} \text { are used in decomposition and reconstruction. The proposed signum of } \\
\text { cosine matrix method is carried out to eliminate the generation of the three }\end{array}$ \\
\hline Keywords: & $\begin{array}{l}\text { matrices at each block and replace it with a signum of cosine matrix. The } \\
\text { proposed signum of cosine matrix is performed faster on the decomposition }\end{array}$ \\
\hline Embedding watermark & and reconstruction. The image is transformed into a coefficient matrix $\mathrm{C}$ using \\
\hline Extracting watermark & $\begin{array}{l}\text { the signum matrix. The } C \text { matrix values are closer to the } S \text { value of singular } \\
\text { value decomposition which can preserve high quality of the watermarked }\end{array}$ \\
\hline Signum cosine matrix & image. The experimental results show that our method is able to produce \\
\hline Singular value & similar imperceptibility and robustness level of the watermarked image with \\
\hline Watermarking & less computational time. \\
\hline
\end{tabular}

This is an open access article under the $\underline{C C B Y-S A}$ license.

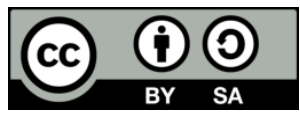

Corresponding Author:

Ferda Ernawan

Faculty of Computing, College of Computing and Applied Sciences, Universiti Malaysia Pahang

Pekan, Pahang, Malaysia

Email: ferda@ump.edu.my

\section{INTRODUCTION}

Image watermarking becomes one of the most rapid-growing fields in computer science. It was driven by the high demand for copyright protection in the current industrial revolution [1], [2]. A watermarking algorithm must have a good level of imperceptibility, strong robustness, and able to work in a short time in order to get a reliable watermarking model. One of the most popular algorithms is singular value decomposition [3]-[6] which has good robustness against compression and filtering but requires a complex mathematical operation for the decomposition and reconstruction. On the other hand, the Chinese remainder theorem model [7], [8] has a good imperceptibility with less complexity but weak against most of the watermark attacks. Currently, the Chinese remainder is mostly developed in secret image sharing [9], [10]

Some models are developed to improve the performance through transformation domain models such as cosine transform [11]-[13], wavelet transform [14]-[16], contourlet transform [17], [18] and even using an optimazion algorithm [19] with the consequences of an increase in processing time. Whereas, the current need is a model with fast computational time, good robustness, and imperceptibility [20]-[22]. Combining several algorithms is not the best solution in optimizing imperceptibility, robustness, or processing time [23].

Generally, there is an inversely proportional relationship requirements of imperceptibility, robustness, and running time. The main challenge is to improve one of the requirements and keeping the others. This paper is proposed a new watermarking method using the signum of cosine matrix which able to replace the singular matrix model from [24], [25] which has three matrices with a single matrix. It decomposes an image into matrices of $U, S$, and $V$. The diagonal matrix $S$ is used in watermark embedding, while the $U$ and $V$ are used in 
the reconstruction process. Meanwhile, the proposed model has used a signum cosine matrix on both decomposition and reconstruction processes to reduce the complexity and faster the processing time.

\section{RESEARCH METHOD}

The main idea of our method is using the largest value of signum of matrix. It is used to embed and extract the watermark bits using a partition function. The detailed explanation of the method is presented in the following section.

\subsection{Signum of cosine matrix}

The signum of the cosine matrix is a signed value of the real numbers from the cosine matrix [26]. For a square matrix of size of $N$, it is as (1):

$$
D(x, y)=\left\{\begin{array}{cl}
\operatorname{sgn}\left\{\sqrt{\frac{1}{N}}\right\} & , x=0 \\
\operatorname{sgn}\left\{\sqrt{\frac{2}{N}} \cos \frac{\pi(2 y+1) x}{2 N}\right\} & , x \neq 0
\end{array}\right.
$$

where $x$ and $y$ are rows and columns, $\operatorname{sgn}\{\cdot\}$ is a signum function defined as (2):

$$
\operatorname{sgn}(d)=\left\{\begin{array}{cc}
1 & , d>0 \\
0 & , d=0 \\
-1 & , d<0
\end{array}\right.
$$

the matrix consists of a signed value of 1 which has a low computational cost. It can be utilized to decompose $n$-th block of host image $I_{n}$ into coefficient matrix $C_{n}$ as (3):

$$
C_{n}=D * I_{n} * D^{-1}
$$

the reconstruction is obtained with:

$$
I_{n}=D^{-1} * C_{n} * D
$$

hence, the decomposition and reconstruction can be done using a matrix of $D$. The method works simpler than the decomposition of singular value which divides image $I_{n}$ into matrices of $U_{n}, S_{n}, V_{n}$. And then reconstructs the image using:

$$
I_{n}=U_{n} * S_{n} * V_{n}^{-1}
$$

the embedding is performed on the largest coefficient of matrix $C$ because it has similar magnitudes with the largest value of matrix $S$. Their largest value is the first element of the matrix which contains the image characteristic. The comparison between the largest value of matrix $S$ and matrix $C$ from random sequence blocks are presented in Figure 1.

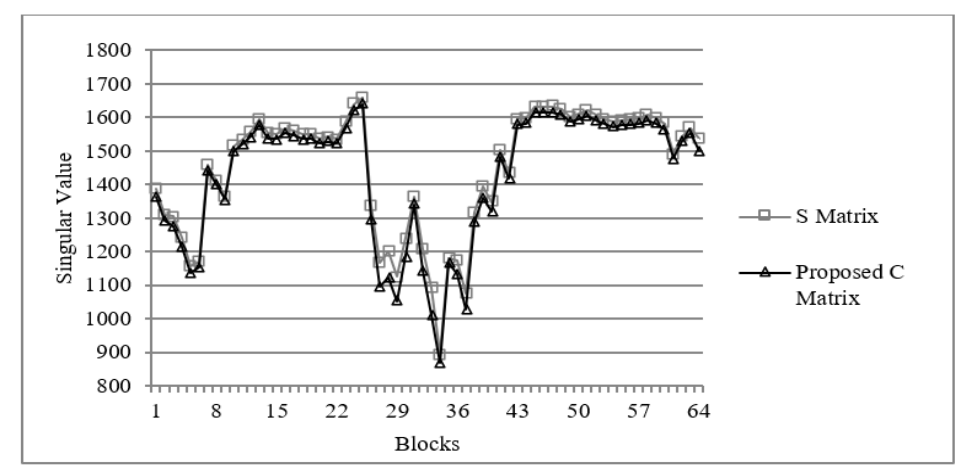

Figure 1. Comparison between largest value of $\mathrm{S}$ matrix and $\mathrm{C}$ matrix from random sequence blocks 
It shows that the largest value of matrix $C$ from (3) is similar to diagonal matrix $S$. The proposed matrix is able to produce a similar value with a simpler model. Moreover, matrix $D$ only needs to be generated once, and then it is used to get the entire matrix $C$, while the $\mathrm{S}$ matrix must perform at each block to get the whole matrix $S$ as seen in (4) and (5). The reconstruction of the $S$ matrix requires $U_{n}$ and $V_{n}$ on each $n$-th block, while the proposed method only requires matrix $D$ on all blocks.

\subsection{Watermark embedding}

The proposed method is intended to reduce the complexity of the $\mathrm{S}$ matrix-based model. Instead of using three matrices, a signum matrix $D$ is use for embedding and extraction as shown in Figure 2. The watermark embedding requires a signum of cosine matrix, partition function, and embedding rules in detail as:

Step 1: Generate a signum of cosine matrix $D$ with the size of $N$ using (1).

Step 2: Split host image $I$ into the block of $N$.

Step 3: Calculate the coefficient matrix $C_{n}$ on each block using as (3).

Step 4: Take the largest coefficient which is the initial value of $C_{n}$ as $c_{n}$.

Step 5: Create equations to divide the value as follows:

$$
\begin{aligned}
& r=(\max (c)-\min (c)) / l \\
& t_{i}=\min (c)+r(i-1) \\
& b_{i}=\min (c)+r(i-2) \\
& m_{i}=\left(t_{i}+b_{i}\right) / 2
\end{aligned}
$$

where $r$ and $l$ are the range and level of partition, which is 40 , while $t_{i}, b_{i}, m_{i}$, are the top, bottom, and middle value of $i$-th partition level.

Step 6: Assign $c_{n}$ to the $i$-th partition using:

$$
b_{i} \leq c_{n}<t_{i}
$$

Step 7: Embed the watermark bit $w$ to get the embedded value $c_{n}^{\prime}$ according to the following condition:

$$
c_{n}^{\prime}= \begin{cases}\left(t_{i}+m_{i}\right) / 2 & , \text { if } w_{n}=0 \\ \left(b_{i}+m_{i}\right) / 2 & \text {, if } w_{n}=1\end{cases}
$$

Step 8: Update the first element of $C_{n}$ using $c_{n}^{\prime}$ to get the embedded matrix $C_{n}^{\prime}$, and then reconstruct the watermarked block $I_{n}^{\prime}$ using as (4).

Step 9: Repeat step 6 until 8 to the remaining blocks to get a complete watermarked image.

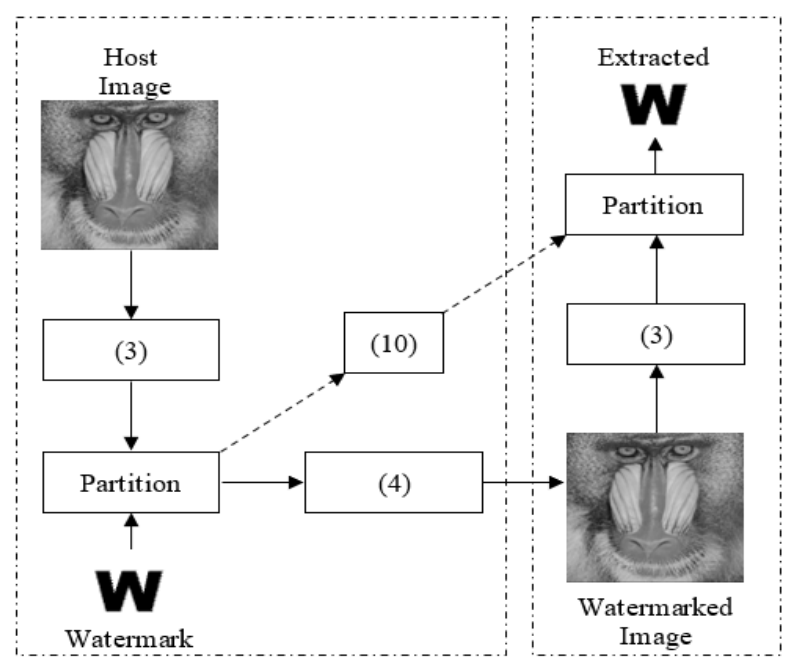

Figure 2. The proposed watermarking scheme 


\subsection{Watermark extraction} is follows:

This process requires a definite extraction rule with the same partition function. The detailed process

Step 1: Split watermarked image $I$ ' into blocks of $N$.

Step 2: Calculate the embedded matrix $C_{n}^{\prime}$ using as (3).

Step 3: Take the embedded value $c_{n}^{\prime}$ from $C_{n}^{\prime}$, and assign the value using as (10).

Step 4: Get the watermark bits using the following rule:

$$
w_{n}= \begin{cases}1 & , \text { if } b_{i} \leq c_{n}^{\prime}<m_{i} \\ 0 & , \text { if } m_{i} \leq c_{n}^{\prime}<t_{i}\end{cases}
$$

Step 5: Repeat step 3 and 4 to get a complete watermark image.

\section{RESULTS AND DISCUSSION}

The experiments were carried out with a standard image dataset as the host to ensure the validity of the test results. Five 8-bit images a with a size of 512x512 are used as host images while a 1-bit image of 64x64 is used as a watermark. The dataset is presented in Figure 3.

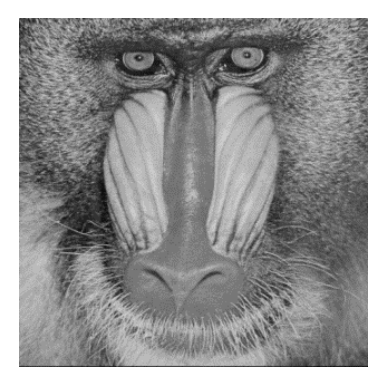

Baboon

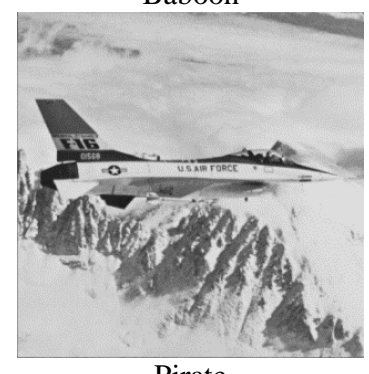

Pirate

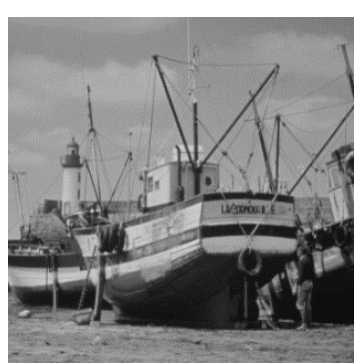

Boat

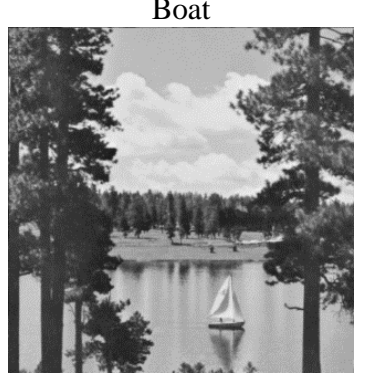

Sailboat

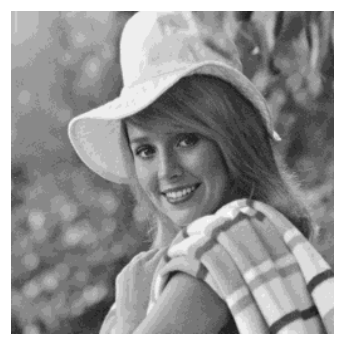

Elaine

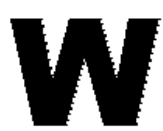

Watermark

Figure 3. Dataset images

\subsection{Imperceptibilitiy and robustness}

The host image that has been embedded with a watermark will experience a change in value and visual. The difference between the image before and after embedding is measured with structural similarity (SSIM) [27] which able to measure the similarity and structure of the images [28]. Table 1 shows that our method has the same value as the $S$ matrix-based watermarking with an average SSIM of 0.9944. It is due to the use of the largest coefficient value that has been proven to be able to produce a value close to the singular value as shown in Figure 1.

Table 1. Comparison of SSIM value between SVD and the proposed scheme

\begin{tabular}{lcc}
\hline \multicolumn{1}{c}{ Images } & S Matrix & Proposed \\
\hline Baboon & 0.9978 & 0.9979 \\
Boat & 0.9936 & 0.9937 \\
Elaine & 0.9940 & 0.9940 \\
Jet & 0.9924 & 0.9924 \\
Sailboat & 0.9940 & 0.9941 \\
Average & 0.9944 & 0.9944 \\
\hline
\end{tabular}


Table 2. Comparison of extracted watermark images

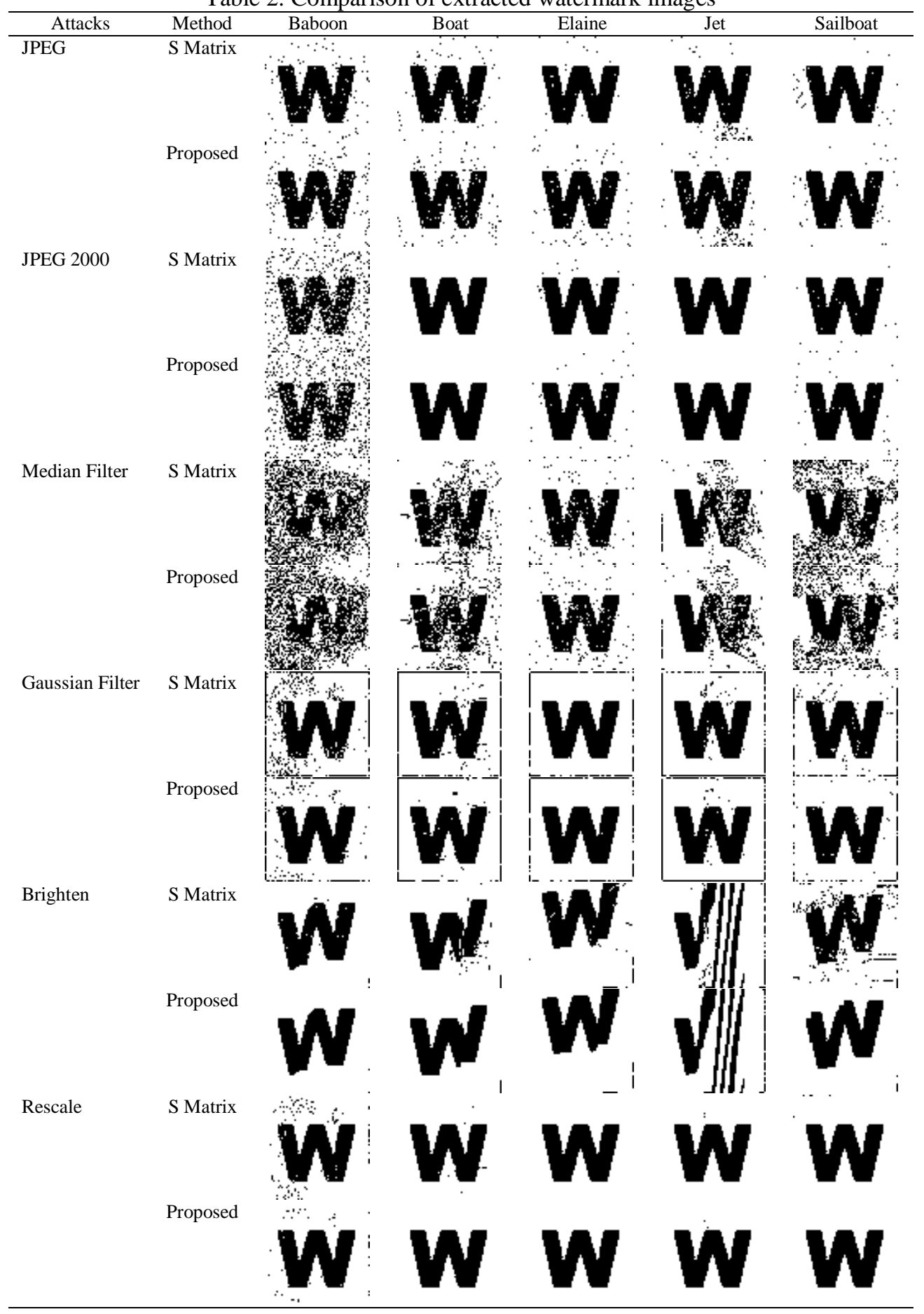

In the next phasewatermarked images are tested using standardized tests that comply with the principles of robustness testing. They are joint photograph expert group (JPEG) with a quality of 50\%, JPEG2000 with a ratio of 5, Gaussian filtering with a filter of $3 \times 3$, and sigma of 0.5 , brightening of 10 , and the rescaling of (2 0.5). Each extracted watermark is compared with the embedded watermark and then meassured normalized correlation (NC) as follows [29], [30].

$$
N C=\frac{\sum_{n=1}^{N} w_{n} w_{n}^{\prime}}{\sqrt{\sum_{n=1}^{N} w_{n}^{2}} \sqrt{\sum_{p=n}^{N} w_{n}^{\prime 2}}}
$$


Where $w_{n}$ and $w_{n}$ are the embedded and extracted watermark from the attacked image of an $n$-th element. The visual comparison of extracted watermark shows in Table 2. Meanwhile the comparison of the NC value is presented in Table 3.

Table 3. Comparison of NC value of extracted watermark

\begin{tabular}{lcccccc}
\hline \multicolumn{1}{c}{ Attacks } & Method & Baboon & Boat & Elaine & Jet & Sailboat \\
\hline JPEG & S Matrix & 0.9809 & 0.9874 & 0.9958 & 0.9863 & 0.9941 \\
& Proposed & 0.9673 & 0.9778 & 0.9850 & 0.9791 & 0.9919 \\
JPEG2000 & S Matrix & 0.9106 & 1.0000 & 0.9963 & 0.9998 & 0.9965 \\
& Proposed & 0.8982 & 1.0000 & 0.9941 & 1.0000 & 0.9956 \\
Median Filter & S Matrix & 0.7362 & 0.9227 & 0.9762 & 0.9303 & 0.8522 \\
& Proposed & 0.7440 & 0.9212 & 0.9752 & 0.9296 & 0.8656 \\
Gaussian Filter & S Matrix & 0.9184 & 0.9531 & 0.9685 & 0.9563 & 0.9660 \\
Brighten & Proposed & 0.9599 & 0.9587 & 0.9674 & 0.9578 & 0.9802 \\
& S Matrix & 0.9361 & 0.9480 & 0.7685 & 0.8257 & 0.8479 \\
Reshape & Proposed & 0.9378 & 0.9532 & 0.7702 & 0.8277 & 0.8791 \\
& S Matrix & 0.9805 & 0.9978 & 1.0000 & 0.9997 & 0.9992 \\
& Proposed & 0.9929 & 0.9997 & 1.0000 & 0.9997 & 1.0000 \\
\hline
\end{tabular}

Tables 2 and 3 show that extracted watermarks from the proposed method have a similar quality to the S matrix with the average NC value of 0.9470 and 0.9444 . Most of the extracted watermarks can be seen properly and have an NC value close to 1 . The use of the largest coefficient value is able to replace the $S$ value. It is only slightly changed even though the watermarked image has experienced significant differences from various attacks.

\subsection{Processing time}

The main purpose of the proposed method is to reduce computational cost of the $S$ matrix. Measurements are performed on the embedding of watermarked image and extraction of the entire attacked watermarked images in seconds. Figure 4 shows that our method is faster with an average time of 0.5327 seconds than the $S$ matrix with a processing time of 0.7513 seconds. As well as in extraction time, the method has an average extraction time of 0.1975 seconds which is faster than the $S$ matrix which has 0.2684 seconds as shown in Figure 5. The method has faster extraction and embedding time compared to the $S$ matrix. It is replaced the decomposition and reconstruction of $U, S$, and $V$ matrices on each block using the coefficient matrix to build a simpler matrix operation.

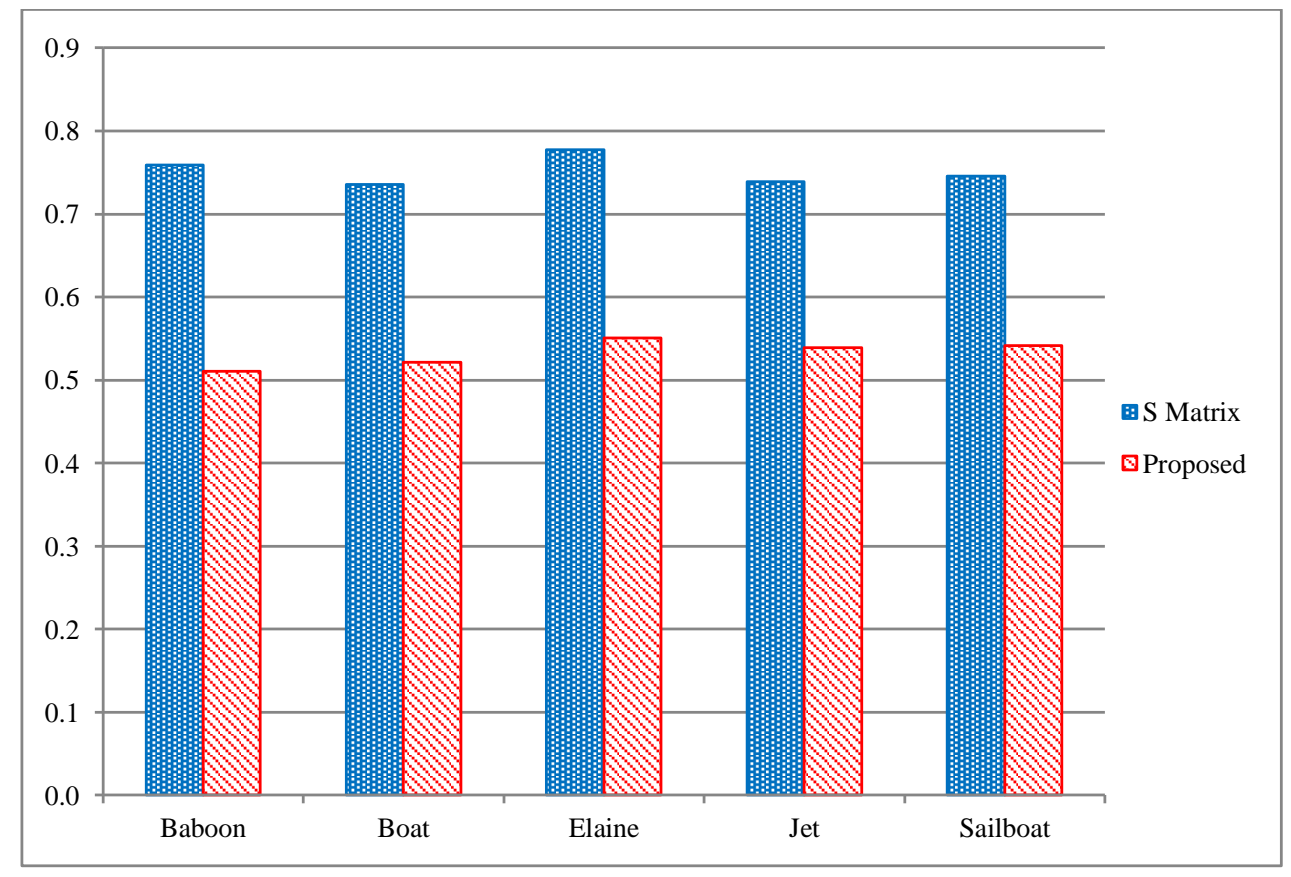

Figure 4. Computational time of the embedding watermark 


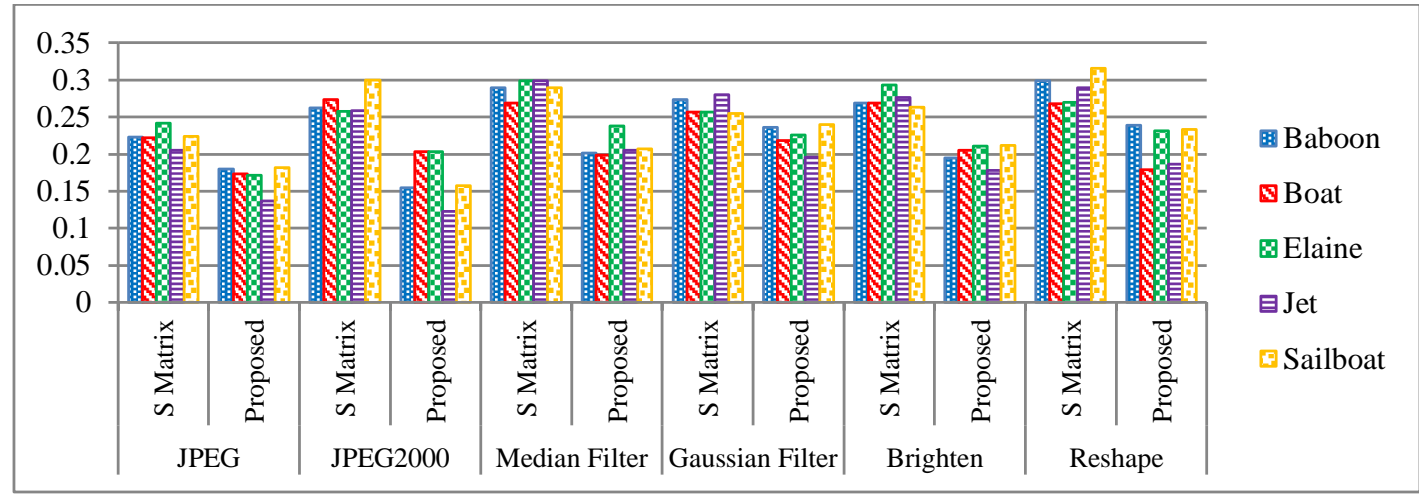

Figure 5. Comparison of extraction time

\section{CONCLUSION}

The proposed signum of cosine matrix method has been used to replace matrices of $U, S$, and $V$ in image watermarking. It is used to generate $C$ matrix which has similar with singular value of singular value decomposition (SVD). The decomposition and reconstruction of singular matrix requires a large computational time on each image block. The proposed signum matrix performs faster than the SVD in image watermarking. The experimental results show that the proposed method produces an average NC value of about 0.9444 under various attacks. The proposed scheme achieves high SSIM value of about 0.9944. In addition, the proposed signum of cosine matrix produces a shorter computational time than the SVD method in terms of embedding and extracting watermark image. The proposed signum of cosine matrix consumes 0.5327 second for embedding watermark and 0.1975 second for extracting watermark. The proposed method performs faster computational time than SVD method in image watermarking with maintaining imperceptibility and robustness performances.

\section{ACKNOWLEDGEMENT}

This work was supported by Fundamental Research Grant Scheme (FRGS), Ministry of Education under Grant No. FRGS/1/2018/ICT04/UMP/02/2, RDU190117 and PGRS1903186.

\section{REFERENCES}

[1] V. M. Manikandan and V. Masilamani, "Histogram shifting-based blind watermarking scheme for copyright protection in 5G," Computers and Electrical Engineering, vol. 72, pp. 614-630, Nov. 2018, doi: 10.1016/j.compeleceng.2018.03.007.

[2] W. Keat Lee and S. Chui n Liew, "Fingerprint Watermarking With Tamper Localization and Exact Recovery Using Multi-Level Authentication," International Journal of Software Engineering and Computer Systems, vol. 4, no. 1, pp. 120-132, Feb. 2018, doi: 10.15282/ijsecs.4.1.2018.10.0043.

[3] H. S. Devi and K. M. Singh, "Red-cyan anaglyph image watermarking using DWT, Hadamard transform and singular value decomposition for copyright protection," Journal of Information Security and Applications, vol. 50, p. 102424, Feb. 2020, doi: 10.1016/j.jisa.2019.102424.

[4] S. Wang et al., "Optical image watermarking based on singular value decomposition ghost imaging and lifting wavelet transform," Optics and Lasers in Engineering, vol. 114, pp. 76-82, Mar. 2019, doi: 10.1016/j.optlaseng.2018.10.014.

[5] T. K. Araghi, A. A. Manaf, and S. K. Araghi, "A secure blind discrete wavelet transform based watermarking scheme using twolevel singular value decomposition," Expert Systems with Applications, vol. 112, pp. 208-228, Dec. 2018, doi: 10.1016/j.eswa.2018.06.024.

[6] S. L. Jia, "A novel blind color images watermarking based on SVD," Optik, vol. 125, no. 12, pp. 2868-2874, Jun. 2014, doi: 10.1016/j.ijleo.2014.01.002.

[7] J. C. Patra, A. Karthik, and C. Bornand, "A novel CRT-based watermarking technique for authentication of multimedia contents," Digital Signal Processing: A Review Journal, vol. 20, no. 2, pp. 442-453, Mar. 2010, doi: 10.1016/j.dsp.2009.07.004.

[8] P. W. Adi, Y. P. Astuti, and E. R. Subhiyakto, "Imperceptible image watermarking based on chinese remainder theorem over the edges," in International Conference on Electrical Engineering, Computer Science and Informatics (EECSI), Sep. 2017, vol. 2017December, doi: 10.1109/EECSI.2017.8239117.

[9] X. Yan, Y. Lu, L. Liu, J. Liu, and G. Yang, "Chinese remainder theorem-based two-in-one image secret sharing with three decoding options," Digital Signal Processing: A Review Journal, vol. 82, pp. 80-90, Nov. 2018, doi: 10.1016/j.dsp.2018.07.015.

[10] M. Deshmukh, N. Nain, and M. Ahmed, "A novel approach for sharing multiple color images by employing Chinese Remainder Theorem," Journal of Visual Communication and Image Representation, vol. 49, pp. 291-302, Nov. 2017, doi: 10.1016/j.jvcir.2017.09.013.

[11] Z. Yuan, D. Liu, X. Zhang, and Q. Su, "New image blind watermarking method based on two-dimensional discrete cosine transform," Optik, vol. 204, p. 164152, Feb. 2020, doi: 10.1016/j.ijleo.2019.164152.

[12] M. Y. Nejad, M. Mosleh, and S. R. Heikalabad, "A blind quantum audio watermarking based on quantum discrete cosine transform,” Journal of Information Security and Applications, vol. 55, p. 102495, Dec. 2020, doi: 10.1016/j.jisa.2020.102495. 
[13] S. Roy and A. K. Pal, “A blind DCT based color watermarking algorithm for embedding multiple watermarks,” AEU - International Journal of Electronics and Communications, vol. 72, pp. 149-161, Feb. 2017, doi: 10.1016/j.aeue.2016.12.003.

[14] Y. Luo et al., "A multi-scale image watermarking based on integer wavelet transform and singular value decomposition," Expert Systems with Applications, vol. 168, p. 114272, Apr. 2021, doi: 10.1016/j.eswa.2020.114272.

[15] F. Ernawan and D. Ariatmanto, "Image watermarking based on integer wavelet transform-singular value decomposition with variance pixels," International Journal of Electrical and Computer Engineering, vol. 9, no. 3, pp. 2185-2195, Jun. 2019, doi: 10.11591/ijece.v9i3.pp2185-2195.

[16] N. Sangeetha and X. Anita, "Entropy based texture watermarking using discrete wavelet transform," Optik, vol. 160, pp. 380-388, May 2018, doi: 10.1016/j.ijleo.2018.01.136.

[17] A. Najih, S. A. R. Al-Haddad, A. R. Ramli, S. J. Hashim, and M. A. Nematollahi, "Digital image watermarking based on angle quantization in discrete contourlet transform," Journal of King Saud University - Computer and Information Sciences, vol. 29, no. 3, pp. 288-294, Jul. 2017, doi: 10.1016/j.jksuci.2016.02.005.

[18] S. Etemad and M. Amirmazlaghani, "A new multiplicative watermark detector in the contourlet domain using t Location-Scale distribution,” Pattern Recognition, vol. 77, pp. 99-112, May 2018, doi: 10.1016/j.patcog.2017.12.006.

[19] A. M. Abdulazeez, D. M. Hajy, D. Q. Zeebaree, and D. A. Zebari, "Robust watermarking scheme based LWT and SVD using artificial bee colony optimization," Indonesian Journal of Electrical Engineering and Computer Science, vol. 21, no. 2, pp. 12181229, Jan. 2020, doi: 10.11591/ijeecs.v21.i2.pp1218-1229.

[20] C. P. Wang, X. Y. Wang, and Z. Q. Xia, "Geometrically invariant image watermarking based on fast Radial Harmonic Fourier Moments," Signal Processing: Image Communication, vol. 45, pp. 10-23, Jul. 2016, doi: 10.1016/j.image.2016.03.007.

[21] S. Bravo-Solorio, F. Calderon, C. T. Li, and A. K. Nandi, "Fast fragile watermark embedding and iterative mechanism with high self-restoration performance," Digital Signal Processing: A Review Journal, vol. 73, pp. 83-92, Feb. 2018, doi: 10.1016/j.dsp.2017.11.005.

[22] H. ying Yang, S. ren Qi, P. pan Niu, and X. yang Wang, "Color image zero-watermarking based on fast quaternion generic polar complex exponential transform," Signal Processing: Image Communication, vol. 82, p. 115747, Mar. 2020, doi: 10.1016/j.image.2019.115747.

[23] P. W. Adi and P. Arsiwi, "A novel watermarking method using hadamard matrix quantization," Journal of ICT Research and Applications, vol. 14, no. 1, pp. 1-15, Jul. 2020, doi: 10.5614/itbj.ict.res.appl.2020.14.1.1.

[24] B. C. Mohan and S. S. Kumar, "A robust image watermarking scheme using singular value decomposition," Journal of Multimedia, vol. 3, no. 1, pp. 7-15, May 2008, doi: 10.4304/jmm.3.1.7-15.

[25] P. W. Adi and F. Z. Rahmanti, "Robust Integer Haar Wavelet Based Watermarking Using Singular Value Decomposition," Jurnal Ilmu Komputer dan Informasi, vol. 9, no. 1, p. 26, Feb. 2016, doi: 10.21609/jiki.v9i1.363.

[26] T. I. Haweel, "A new square wave transform based on the DCT," Signal Processing, vol. 81, no. 11, pp. 2309-2319, Nov. 2001, doi: 10.1016/S0165-1684(01)00106-2.

[27] Z. Wang, A. C. Bovik, H. R. Sheikh, and E. P. Simoncelli, "Image quality assessment: From error visibility to structural similarity," IEEE Transactions on Image Processing, vol. 13, no. 4, pp. 600-612, Apr. 2004, doi: 10.1109/TIP.2003.819861.

[28] A. Nagm and M. Safy, "A robust watermarking algorithm for medical images," Indonesian Journal of Electrical Engineering and Computer Science, vol. 20, no. 3, pp. 1601-1612, Dec. 2020, doi: 10.11591/ijeecs.v20.i3.pp1601-1612.

[29] F. Ernawan, "Tchebichef image watermarking along the edge using YCoCg-R color space for copyright protection," International Journal of Electrical and Computer Engineering, vol. 9, no. 3, pp. 1850-1860, Jun. 2019, doi: 10.11591/ijece.v9i3.pp1850-1860.

[30] P. W. Adi, F. Ernawan, A. Wibowo, E. A. Sarwoko, and F. Agung Nugroho, "Watermarking Scheme based on Chinese Remainder Theorem and Integer Wavelet Filters for Copyright Protection," in Proceedings - 2021 International Conference on Software Engineering and Computer Systems and 4th International Conference on Computational Science and Information Management, ICSECS-ICOCSIM 2021, Aug. 2021, pp. 70-74, doi: 10.1109/ICSECS52883.2021.00020.

\section{BIOGRAPHIES OF AUTHORS}

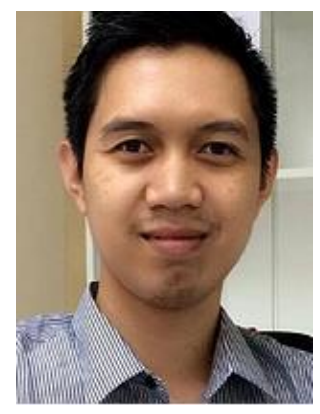

Ferda Ernawan (iD 8) SC P is currently a Senior Lecturer at the Faculty of Computing, College of Computing and Applied Sciences, Universiti Malaysia Pahang. He received his Master in Software Engineering and Intelligent and Ph.D from the Faculty of Information and Communication Technology, Universiti Teknikal Malaysia Melaka in 2011 and 2014 respectively. His research interests include image compression, digital watermarking and steganography. He can be contacted at email: ferda@ump.edu.my.

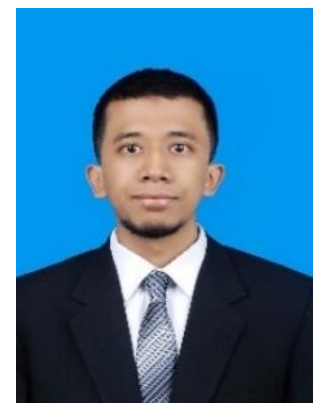

Prajanto Wahyu Adi (D) SC S received the Barchelor in computer science form Universitas Stikubank, Indonesia in 2011. He finished his master's degree through a dual degree program in information technology at Universitas Dian Nuswantoro, Indonesia and in field of computer science at the Universiti Teknikal Malaysia Melaka, Malaysia in 2014. He served as a lecturer at the Department of Information Techology, Faculty of Computer Science, Universitas Dian Nuswantoro and currently works as a lecturer at the Department of Informatics, Faculty of Science and Mathematics, Universitas Diponegoro. His areas of expertise are cryptography, digital image classification, steganography and watermarking. He can be contacted at email: prajanto@lecturer.undip.ac.id. 

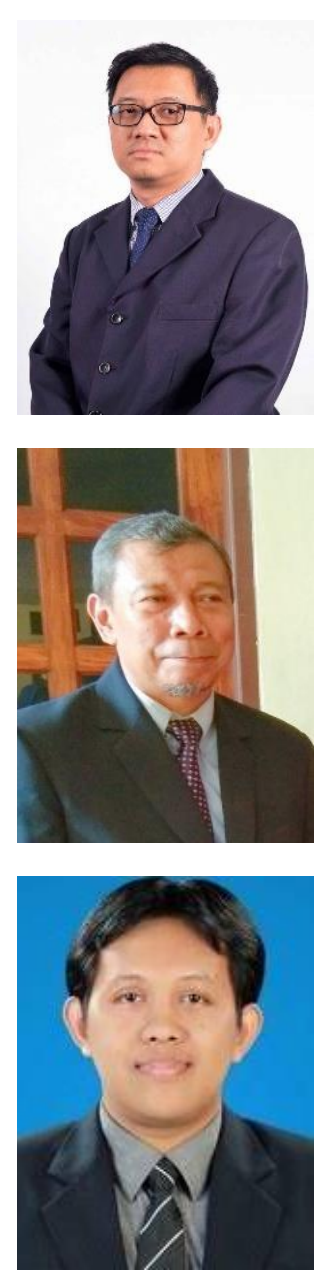

Siau-Chuin Liew (D) 8 SC P is currently a Senior Lecturer at the Faculty of Computing, College of Computing and Applied Sciences, Universiti Malaysia Pahang. He received his Master in Strategic Business IT from University of Portsmouth in 2006 and Ph.D in Computer Science from Universiti Malaysia Pahang in 2011. His research interests include biomedical image processing. He can be contacted at email: liewsc@ump.edu.my.

Eko Adi Sarwoko (D) 8d SC P received the Barchelor degree in mathematics from Unviersitas Diponegoro, Indonesia in 1991 and the Master of computer science at Universitas Indonesia in 2003. He works as a lecturer at the Department of Informatics, Faculty of Science and Mathematics, Universitas Diponegoro. He has served as Head of the Department of Informatics in 2009 - 2014, Universitas Diponegoro and was on the board of directors of the Association of Informatics and Computer Science Colleges (Aptikom) in 2016-2021. His research fields include deep learning, information hiding, object detection and classification. He can be contacted at email: ekoadisarwoko@ lecturer.undip.ac.id.

Edy Winarno (iD) 8 SC $\mathrm{P}$ received the Doctoral degree in Computer Vision from Department of Computer Science, Faculty of Mathematics and Natural Sciences, Gadjah Mada University Indonesia 2011-2015. He is a lecturer in Informatics Engineering Department, Stikubank University Indonesia. His research area in Computer Vision, image processing and artificial intelligent. He has authored or coauthored more than 25 Scopus indexed publication, with $6 \mathrm{H}$-index. His research interest includes pattern recognition, cryptography, expert system and intelligent system. He can be contacted at email: edywin@edu.unisbank.ac.id. 